\title{
Antecedents of achalasia
}

Since I first read Sir Arthur Hurst's Essays and Addresses ${ }^{1}$ as a student, achalasia of the cardia has been fascinating me for more than 50 years. Hurst had trained as a neurologist and, with G W Rake, ${ }^{2}$ clearly identified achalasia as a neurological disorder by showing depletion of ganglion cells in the oesophageal myenteric plexus. In addition they found inflammatory changes with lymphocytic infiltration in the plexus. Although degenerative changes have since been reported ${ }^{3}$ in the dorsal vagal nucleus and in the vagus nerve, unlike those in the oesophageal myenteric plexus, these have never been substantiated. Histological examination showed few further clues until 40 years later when, classifying ganglion cells according to their affinity for silver stains, Barbara $S$ mith ${ }^{4}$ noted that it was the argyrophil cells that were lacking. These cells, which have several axons and many dendrites, have been considered the organising cells and probably those mediating inhibition of smooth muscle. In contrast, the argyrophobe cells, mediating muscle contraction through the release of acetyl choline, were comparatively unaffected in achalasia.

Physiological studies in the last 50 years have established the absence of peristalsis in the lower oesophagus and failure of relaxation of the often hypertensive lower oesophageal sphincter, which are compatible with a lesion in the oesophageal myenteric plexus rather than the dorsal vagal nucleus or the vagus nerve. Evidence of vagal impairment in achalasia remains equivocal; at endoscopy a few patients have a gastric food residue and delayed gastric emptying has been reported in those examined after pneumatic bag dilatation, yet symptomatic motility disorder in the gut below the cardia is almost unknown in achalasia.

The gastric acid secretory response to insulin has been variously reported as subnormal in up to $50 \%$ of patients with achalasia. Using the insulin/pentagastrin secretory ratio, which makes allowance for parietal cell mass, one study reported an abnormality in $30 \%$ of patients with achalasia ${ }^{5}$ but in another no abnormality was found. ${ }^{6}$ Sham feeding has also showed a subnormal gastric acid output in some patients. ${ }^{7}$ Inconclusive as these findings are, it is important to recall that while conventionally used as a measure for the intactness of the vagal trunk, such tests would be expected to show abnormality if the lesion were in the gastric myenteric plexus. Unfortunately no detailed histological studies of the gastric Meissner and Auerbach plexuses in achalasia are available. Other tests that are dependent upon the intact vagus, such as the contraction of the lower oesophageal sphincter with increase of intraabdominal pressure and pulse rate variability with respiration, have given normal results in achalasia. ${ }^{6}$ Clinical pharmacological studies, ${ }^{8}$ and in vitro studies on smooth muscle fibres from the lower oesophageal sphincter both confirm that the cholinergic innervation is intact. Thus there is no convincing histological or pathophysiological evidence of damage to the trunk of the vagus nerve.

While the introduction of new techniques since Hurst's time has helped to delineate the histopathology and pathophysiology of achalasia, remarkably little headway has been made in our understanding of the causation of the disease. One of the important difficulties in identifying the cause of the disease is the long interval between the start of the pathological process and the onset of symptoms. A further problem lies in the inaccessibility of the oesophageal myenteric plexus for histological study, which usually precludes examination early in the course of the disease before evidence of the causative process has disappeared.
Finally, the lack of comparability in the distribution of smooth and striated muscle in the oesophagus between animals and humans means that there is no effective experimental animal model. Any explanation of aetiology must account for the remarkable localisation of the disease to the oesophagus and this virtually excludes a primary lesion in the central nervous system or the vagus nerve. The age of presentation varies from early childhood to middle age or even later. Ischaemic damage to the oesophagus during gut rotation in utero therefore seems unlikely. There is no evidence to suggest that poisons, such as the anthroquinone purgatives, which are known to damage the colonic myenteric plexus, or oesophageal trauma of any kind could be responsible.

The reported occurrence of achalasia in members of the same family ${ }^{9}$ has led to the suggestion that it is an autosomal recessive hereditary disease. A survey, however, of 1012 first degree relatives of 159 patients with achalasia failed to show a single case. ${ }^{10}$ The possibility of siblings being exposed to a common environmental factor seems the most likely explanation of familial incidence but a rare and distinct hereditary childhood disorder cannot be excluded.

The suggestion by Rake and Hurst that the disease has an inflammatory basis led to the speculation that the two common chronic inflammatory diseases of that era, syphilis and tuberculosis, might be involved; one of Hurst's patients had been diagnosed as having tabes dorsalis but his serology, like that of other patients, was negative. In passing Hurst notes that his colleague, C P Symonds, 'suggested that the condition might sometimes be due to an infective ganglionitis analogous to the inflammation of the posterior nerve root ganglion in herpes zoster'.

The striking localisation of the disease to the oesophagus might offer clues to its causation; the oesophagus is the only part of the smooth muscled gut that is lined by squamous epithelium, which confers susceptibility to various infections. Viruses of the herpes group are recognised to have a predilection for squamous mucosa and are known to be neurotropic. Herpes simplex 1 virus, cytomegalovirus, and varicella zoster virus all attack the oesophagus but rarely attack the remainder of the gut. A search for these viruses in the myenteric plexus of the oesophagus, using DNA hybridisation, showed positivity for varicella zoster DNA in $33 \%$ of biopsy specimens taken at the time of cardiomyotomy but all tissue samples from non-achalasic controls proved negative. ${ }^{11}$ Could achalasia, like shingles, be the consequence of a previous attack of chickenpox? Serum complement fixation tests for varicellar zoster virus show a greater positivity in patients with achalasia than control subjects. Furthermore, an association between treated lymphoma and the subsequent development of achalasia has been described ${ }^{12}$ and of course varicella and herpes zoster are also associated with lymphoma and immunoincompetence. This is rather a tenuous link, however, and an explanation is needed for the negativity of DNA probing in two thirds of achalasia patients. The method may be comparatively insensitive and thus fails to detect small amounts of viral DNA or, having caused ganglion cell destruction, the virus has died out.

Although the aetiology of achalasia remains to be established, present evidence points to an inflammatory process, possibly of viral origin, in the oesophageal myenteric plexus. Perhaps the neurologist, Sir Charles Symonds, when he suggested to Hurst that the oesophageal lesion might be 
analogous to that in the posterior nerve root ganglion in herpes zoster, came closer to the mark than have gastroenterologists since.

Department of Surgery, University of Nottingham,

ICHAEL ATKINSON

University Hospital,

Oueen's Medical Centre,

Nottingham NG7 2UH

1 Hurst AF. Essays and addresses on digestive and nervous diseases and on Addison's anaemia and asthma. London: Heinemann, 1924

Rake GW. On the pathology of achalasia. Guy's Hospital Reports 1927; 77; $141-50$

Cassella RR, Brown AL, Sayre GP, Ellis FH Jr. Achalasia of the esophagus: pathologic and etiologic considerations. Ann Surg 1964; 160: 474-86.
4 Smith B. The neurological lesion in achalasia of the cardia. Gut 1970; 11: 388-91.

Elder JB, Gillespie G. The vagus and achalasia. Gut 1969; 10: 1045.

6 Atkinson M, Ogilvie AL, Robertson CS, Smart HL. Vagal function in achalasia. $Q \mathcal{F}$ Med 1987; 63: 297-303.

Raju GS, Rao PN, Broor SL. Effect of sham feeding on gastric acid secretion in patients with achalasia. Gastroenterologv 1989; 96: 989.

8 Holloway RH, Dodds WJ, Helm JF, et al. Integrity of cholinergic innervation to the lower esophageal sphincter in achalasia. Gastroenterologv 1986; 90: $924-9$

9 Westley CR, Herbst JJ, Goldman S, Wisek WC. Infantile achalasia: inherited as an autosomal recessive disorder. $\mathcal{F}$ Pediatr $1975 ; 87: 243-6$

10 Mayberry JF, Atkinson M. A study of swallowing difficulties in first degree relatives of patients with achalasia. Thorax 1985; 40: 391-3.

11 Robertson CS, Martin BAB, Atkinson M. Varicella-Zoster virus DNA in the oesophageal myenteric plexus in achalasia. Gut 1993; 34: 299-302.

12 Benjamin SB, Castell DO. Achalasia and Hodgkin's disease: a chance association? f Clin Gastroenterol 1981; 3: 175-8.

\section{International gastroenterology}

\section{Gastroenterology in China}

With the establishment of New China in 1949, a nationwide educational programme in public health and sanitation was adopted. This was not confined to metropolitan cities and towns; it was even more intensively carried through in rural areas where measures were instituted to control prevalent parasitic diseases. The first important campaign, beginning as early as December 1949, was against schistosomiasis and this showed the wretched conditions then prevailing in rural districts. Our central government emphasised the importance of this campaign by the presence of the minister of public health in person, together with the governors of 13 provinces and one cosmopolitan city (Shanghai), on a leadership board to organise, firstly, the anti-schistosomiasis campaign and, secondly, the eradication of three other important parasitic diseases: kala-azar, filariasis, and malaria and, at the same time, to improve sanitation in the countryside. It was this board that rapidly appreciated the extent to which the vast farming districts suffered from a dire shortage of medical personnel. It was clear that this could not be remedied by relocation of the medical practitioners who were, at that time, mostly working in large cities, and it had become obvious that the small numbers of trained doctors were unequal to the task in hand.

Therefore, the board decided to enrol young people, who had graduated from primary and junior middle schools in each of the provinces, to be trained on a one year course on the use of anti-parasitic drugs, mainly against schistosomiasis, and also to survey sanitation facilities. This 'army' of young people was known as the 'barefoot doctors'. It has to be remembered that, in the period between 1950 to 1956 , there was a continuing shortage of well trained doctors especially in the field of parasitic disease and it was this shortage that was filled by the 'barefoot' personnel. There are no more 'barefoot doctors', but the work done by these young people is recognised and honoured to this day. The campaign against parasitic diseases was a success, as judged by a considerable reduction in the prevalence of schistosomiasis, filariasis, and malaria and the near complete eradication of kala-azar.

Before 1975, there was no organised society of gastroenterology in China but gastroenterology, as a speciality, was only recognised as a branch of the Chinese Society of
International Medicine (CSIM). Through the great efforts and wise counsel of the late distinguished Professor XiaoQian Zhang, of Peking Union Medical College, the Chinese Gastroenterology Society was established in 1978. Since then, the Society has flourished and prospered so rapidly that almost every province as well as important cosmopolitan cities in China will have a subsidiary society and one or more institutes or centres that are affiliated to the Society where clinical gastroenterology and research are carried out; membership of the Chinese Gastroenterological Society has now reached a total of about 5000. There is a national assembly every three years to highlight and discuss the work being done in various parts of China. Since 1981, there has been an official journal of the Society named The Chinese Fournal of Digestion, originally a quarterly and now a bimonthly journal. Within the last five years, there have been several successful large international symposia on gastroenterology in China.

The era of modern gastroenterology in China began with the introduction of endoscopy in the 1970s. There are now several endoscopes of Chinese manufacture that have been distributed to large and small hospitals in the nation. Every hospital has a gastrointestinal clinic with a specialist staff and all young specialists are familiar with diagnostic upper gastrointestinal endoscopy. Therapeutic endoscopy is also practised but mainly in the gastroenterology centres in large cities.

Gastrointestinal diseases are much influenced by geography, personal habits, customs, and ethnic origin. In China, some diseases are more prevalent such as chronic gastritis resulting from Helicobacter pylori, gastric cancer, viral hepatitis (especially hepatitis B), and hepatoma but inflammatory bowel disease, colorectal cancer, and pancreatic cancer are less common than in Western countries. Because gastric cancer and hepatoma threatens the lives of so many Chinese people, there is considerable effort by Chinese gastroenterologists on research into the treatment and, more important, the prevention of these malignant tumours.

SHAO-JI JIANG

Shanghai Institute of Digestive Disease,

145 Shan-Dong Zhong Road,

Shanghai 200001

China 